\title{
Blockchain for the Energy Transition
}

\author{
S. Vergura \\ Department of Electrical and information Engineering \\ Polytechnic University of Bari \\ st. E. Orabona, 4, 70125, Bari, ITALY \\ Phone:+0039 080 5963590, e-mail: silvano.vergura@poliba.it
}

\begin{abstract}
The massive impact of the wind and PhotoVoltaic (PV) power on the power systems require new approach and strategies, because of their unpredictability. This large expansion has been due to the feed-in tariffs, financial support and a concentration of R\&D activities. Moreover, the distributed energy sources require a pervasive and complex management of the energy exchanges. Until now, the management has been usually based on a central authority, but new paradigms based on the BlockChain (BC) technology are very promising. This approach can also be useful to manage the peak power as well as the Demand-Response (DR) strategies. The paper presents this challenging tool for the current energy transition, highlighting the intrinsic features of the blockchain technology and the opportunities for the future energy systems, e.g. the execution of smart contracts in Peer-to-Peer (P2P) networks. This approach will become increasingly useful to manage the energy exchanges in new contexts, where the number of prosumers and active users is growing. The paper also proposes an application of the $\mathrm{BC}$ to manage the peak demand, using the energy injected into the grid by the electric vehicles.
\end{abstract}

\section{Keywords}

Blockchain, Distributed ledger technology, Energy Transition, Renewable Energy Sources, P2P exchange.

\section{Introduction}

Wind turbines and PV systems are intermittent energy sources [1]-[2], whose production depends on the weather conditions. This is a criticality for the operation of electrical system, with respect to its stability and safety [3]. Advanced sensors-based strategies are used to mitigate the impact of the Renewable Energy Sources (RES), thanks to fast-acting supply and storage systems [4]. Smart meters used in Europe and based on ICT are introduced in [5]. Moreover, the microgrids [6-8] are challenging solutions to support the development of the energy communities, whereas the smart grids represent a new paradigm to manage the penetration of the PV plants in the distribution lines. The criticalities regard the unpredictability of the produced energy and the efficiency of the plants [9-12]. Another issue linked to the electrical grid regards the energy exchange with the Electric Vehicles (EVs). In fact, the standard use of the electrical charging unit provides the energy flow direction from the grid to the vehicle, in order to charge the onboard battery. Instead, nowadays, some Distributed Systems Operators (DSO) are evaluating the use of the bi-directional charging units, so-called Vehicle-to-Grid (V2G). In this way, the vehicles can inject the energy stored in their batteries into the grid during the peak hours, against an economic fee. In any case, it is mandatory a reliable communication system between the prosumers (the users that can either inject or absorb energy) and the active users (the users who make their load management available). This approach is based on the transactive energy [13], defined as "a system of economic and control mechanisms that allows the dynamic balance of supply and demand across the entire electrical infrastructure using value as a key operational parameter." The value is defined by the price or by the incentives to the prosumers.

These are only some examples of electrical systems that provide an energy exchange between two or more users. Usually, these systems are based on a central management unit that guarantees the reliability and the security of the transactions. An effective modern tool to manage the energy transactions [14]-[15] can be the blockchain that allows to do the transactions directly, avoiding the costs and the times of the intermediaries. The paper presents the fundamentals of the $\mathrm{BC}$ and the applications in the energy sector. Finally, the paper proposes a $\mathrm{BC}$ to manage the peak demand, by using the $\mathrm{V} 2 \mathrm{G}$ approach. The proposal is based on the normalized energy, i.e. the ratio between the energy injected into the grid and the nominal capacity of the battery. This strategy allows every owner of an EV to validate a new block, even if his EV is equipped with a small capacity battery. Section II presents the BC and the main features. Section III discusses the consensus algorithms and the applications of the BC in the energy sector. Section IV proposes an application to manage the peak demand, thanks to the EVs. Conclusions end the paper.

\section{Fundamentals of a blockchain}

\section{A. Distributed Ledger Technology}

The BC is a digital data structure, based on a shared and distributed database, containing a log of transactions, in chronological order. The $\mathrm{BC}$ belongs to the family of the so-called Distributed Ledger Technology (DLT), with the feature that the transactions are chronologically ordered. Therefore, the BC is considered as a DLT. Instead, the 
composed name, block-chain, implies that the BC is constituted by blocks (each one closely linked to the previous one, forming a chain), which contain transactions. Each block is time-stamped and subjected to cryptography, guaranteeing the contents and the creation time. Thus, the $\mathrm{BC}$ can be considered as a digital network, where each transaction is proposed, accepted/rejected, stored and shared among the participants. Fig. 1 reports a principal scheme of how the BC works. Any transition that happens outside the $\mathrm{BC}$ has similar approach, but is based on a third party. The third part represents a trusted part, which has a guarantee role between two conflicting interests. For example, a bank has the role of a third party in a financial transaction and the same role is covered by the Authority of any Utility. These last ones are typical centralized systems (Fig. 2a), which manage the requests and define the transaction conditions between conflicting interests. The presence of the third party requires additional steps in any procedure, and it implies costs.

\section{HOW THE BLOCKCHAIN WORKS}
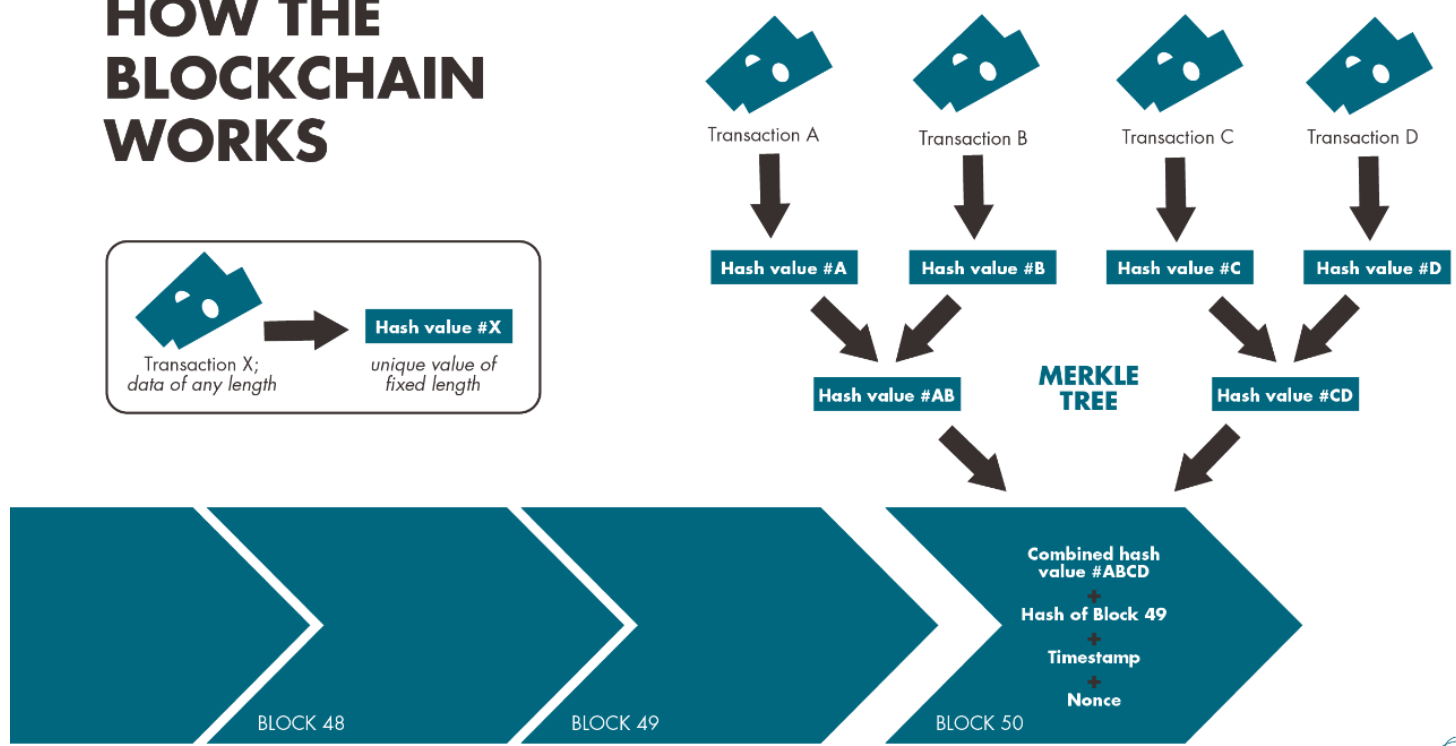

Reproduction of an original figure in "The Great Chain of Being Sure About Things" by the Economist Development

Fig. 1. Principal scheme to create a new block to add in the blockchain. In evidence, the link to the previous block 49, the timestamp, and the nonce (figure under Creative Commons, Attribution-Share Alike 4.0 International, https://commons.wikimedia.org/wiki/File:Blockchain_workflow.png).

Instead, the $\mathrm{BC}$ has not a third party to manage the transactions. The guarantee role is covered by the shared and distributed ledger (Fig. 2b), where each transition is stored and cannot be deleted. So, the BC is a distributed system, without any central management. It is based on the digital users who cooperate to guarantee the reliability of the transactions, the security of the ledger that stores the transactions, and its immutability. Every member holds the updated copy of the ledger, thus the transparency acts as control.

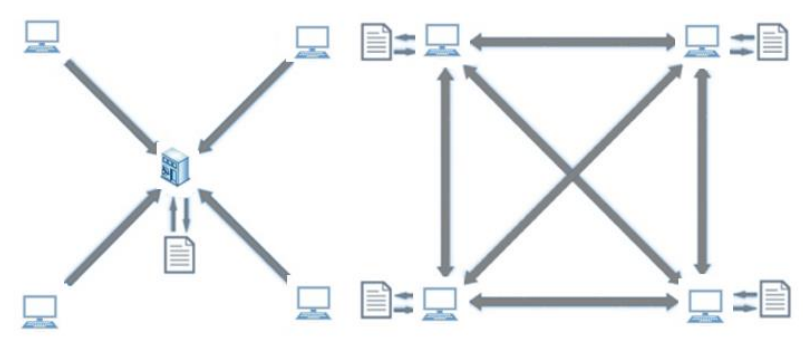

(a)

(b)

Fig. 2. a) centralized system, with an unique ledger; b) distributed system, with as many copies of the ledger as there are users.
The most known BCs are Bitcoin and Ethereum. The first one is the famous crypto-currency, instead the second one is a paradigm for many applications different from the currency. Bitcoin is an electronic payment system, based on P2P anonymous users. The transactions are not verified by a central bank but managed by collaborative computers that assure security, by means of the cryptography. Different from Bitcoin, Ethereum is a cloud $\mathrm{BC}$, based on an embedded programming language that allows users to create new applications. Since it is a programmable $\mathrm{BC}$, the user is not constrained to use standard operations, but he/she can create customized operations for any specific target. This feature of Ethereum is a strength for applications in the energy sector, where a lot of diversified areas are present. For example, Ethereum is used to create smart contracts and user-built platforms for applications based on transactions among the users. Other applications will be discussed in the Section III.

\section{B. Features of the BCs}

The BCs have some main features that allow defining the operating perimeter and the constraints for the users. 
These features are usually defined when the BC is designed, even if some not frequent modifications are possible during its operation. Specific rules in the BCs are represented by the users and the validators. Users are the active parts of the transactions (the sending part and the receiving one), whereas the validators are responsible of the modifications in the ledger. After a modification is complete (by means of the distributed consensus defined in the next section), the modified ledger is available also to the users. Some BCs are configured for an universal access for the users, other ones are configured for a partial access. Some BCs are public, so everyone can take part, whereas other BCs are private and only authorized users can participate. Sometimes, the BC is based on a permissionless ledger, i.e. any participant of the $\mathrm{BC}$ can have an active role in the validation of the transactions; therefore, each user is also a validator. Instead, permissioned ledgers are structured to give privileges for the modifications of the ledger only to specific nodes. In this case, users and validators have different privileges. The validators of private and permissioned ledgers are known and trusted. Finally, the BCs can be based on open-source or closedsource architectures. It is evident that one of the most important features of any $\mathrm{BC}$ is the procedure to validate transactions, which allow adding a new block to the chain. This procedure is known as distributed consensus algorithm, and it will be explained in the next section.

\section{Distributed consensus algorithm}

The distribution system is trusted, because it is based on a common validation process that consolidates the ledger. This process is said Distributed Consensus Algorithm (DCA) and can be different among different BCs. Nevertheless, some DCAs have currently a predominant position, due to the experienced applications. The aim of the consensus algorithm is to generate a new block and to add it to the BC. Since there is no central trusted unit, the consensus requires several steps, which conclude with a cryptographic finalization. The strategies to validate a new block are different, e.g. Proof of Work (PoW), Proof of Stake (PoS), Practical Byzantine Fault Tolerance (PBFT), Proof of Activity (PoAc), Proof of Capacity (PoC), Proof of Authority (PoAu), and so on. Each of them implements a specific strategy to guarantee not only the reliability of the procedure, but also several performance characteristics as the speed, the scalability, and the transaction security. Moreover, the BC must be resilient to failure, corrupted messages, malicious attacks, and so on.

PoW is the largest used DCA (Fig. 3). It is based on validators nodes (also said miners) that compete to solve a cryptographical puzzle. The first one to solve it receives a bonus represented by a financial reward. Each block contains metadata in the header: the hash of the previous block, a hash linked to all the previous blocks, a random number (nonce) and a target value. Each validator seeks resolution, constituting by a valid output lower than the assigned target. When it is found, the validator returns the block to the network and it is definitively added to the BC. The shared ledger guarantees the reliability of the process. In fact, only if at least $51 \%$ of the computational power is corrupted, the written blocks of the BC can be re-written. The described procedure allows understanding that the process is not very fast. For example, Bitcoin can process about 7 transactions/second and 1 block/minute. Another issue regards the electricity consumption. In fact, PoW is known to be energy-intensive, so several alternatives are investigated. Nevertheless, PoW is considered one of the most reliable strategies and it still retains the predominant role among the DCAs. PoW is used in Bitcoin and Ethereum, even if the second one is evaluating to substitute the PoW with the PoS.

PoS does not use the computational work of PoW, but a random selection process, depending on the wealth of the validators. PoS could be the faster than PoW, even if nowadays it has still some specific criticalities. Indeed, many researchers are trying to improve it by reducing the electricity consumption and maintaining the reliability. In fact, PoW is used in public and private $\mathrm{BCs}$, for permission-less and permissioned ledger, and so on. Also in PoS, the participants are stimulated with a reward.

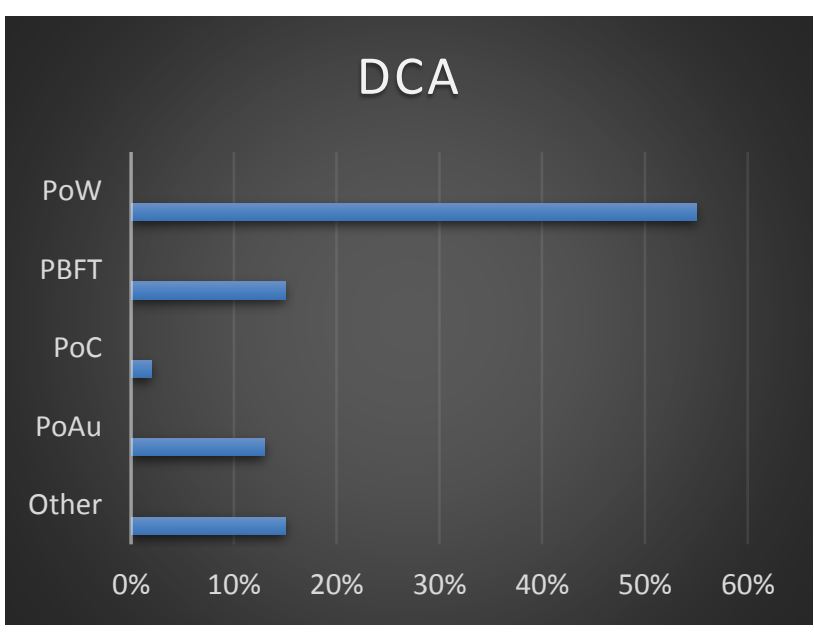

Fig. 3. Popularity of the distributed consensus algorithms.

PBFT is a DCA based on the strategy of a joint action, as the Bizantine generals did during a fortress attack. Joint action implies that all the participants to the attack are informed and the number of traitors, who could make the attack fail, is limited. From the BC point of view, it means that a small number of malicious nodes should not be able to validate a bad block. Some researchers propose that the maximum tolerated traitors must be less than $33 \%$ of the total number.

$\mathrm{PoC}$ is an energy-saving DCA and is based on a cheap hardware. Its name (Proof of Capacity) implies that it is based on database, named plots, which occupies the storage space. Some features are similar to those discussed in PoS.

PoAu is a modified version of PoS, based on special authorization for one or more members who can update the blockchain, generating a new block. This DCA has a constraint to add a new block; in fact, it requires that a new block can be accepted, only if it has been signed by most validators. An implemented voting system allows adding new validators, i.e. authorized nodes. This DCA, based on permissioned validators, has a centralized structure, typical for regulatory body or public administrations. Some utility companies in the energy sector, e.g. Energy Web blockchain, are experimenting this DCA. 
In summary, BC technology is a useful support and guarantees better results than other approaches, when most of the following conditions are met:

- common database;

- multiple parties involved;

- $\quad$ arties with conflicting interests;

- uniform rules for the participants;

- stable rules in the time;

- immutable log.

\section{DCAs and applications in the energy sector}

As seen in the previous section, BC is an effective and trusted tool to manage transactions without any intermediary. It is not relevant what the object of the transaction is: monetary, energy or another one. Moreover, the energy sector is nowadays constituted by many different areas, and each of them has specific characteristics. For example, applications of BCs in the energy sector are already available for the wholesale energy trading, the imbalance settlement, the Internet of Things (IoT) platforms for smart home applications, the distributed energy and the P2P trading, the market model for the trading carbon emissions or green certificates, and so on. Fig. 4 reports the popularity of the most common $\mathrm{BCs}$ for the energy sector and Ethereum, as already said, results largely the most used one. Nevertheless, this predominance depends also on the consideration that it is one of the first BCs. Another strength is that it is not as energy intensive as Bitcoin.

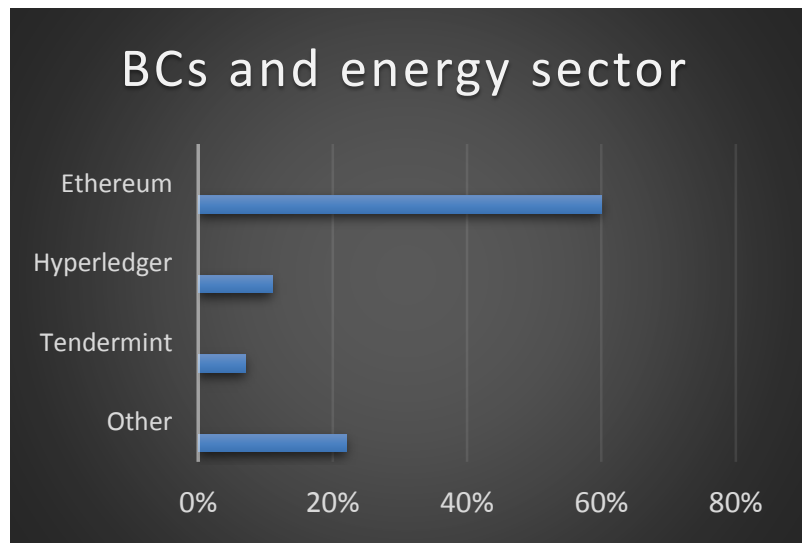

Fig. 4. BCs used in the energy sector.

Let us analyse the case of end-users who participate to the energy market. It is known that nowadays there exist a lot of prosumers that have installed PV plants, wind turbines and other typologies of RES. The prosumers could be interested to directly participate in the energy market, whereas their participation is not allowed nowadays, because they can only sell the produced energy to a central manager of the utility. It is well known that the utility companies purchase this energy at lower price than the standard tariff and the difference represents their revenue. Instead, by using the $\mathrm{BC}$, the prosumer could sell the extra energy to the end-consumers, receiving the related payment. In this way, the transaction would be direct, and the costs could be lower than those paid with a central management unit, but not null. In fact, the exchanged energy will use the electrical infrastructure constituted by the network, the sensors, the protection devices, etc., whose owners are not the sellers neither the buyers.

Another valuable application of the $\mathrm{BC}$ regards the emobility that includes a lot of parties: vehicles, drivers, charging stations, sharing services, and so on. Also in this case, the decentralized infrastructure (e.g. the charging station) could substitute the actual centralized infrastructure, making the transactions competitive and reducing the price of a single charge. The price could be related to other ancillary services of the DSO and could be variable, depending on the presence/absence of the peak demand. E.g., this strategy could be inserted in a V2G approach and the end-users could receive economic rewards. There are already applications for this purpose; for example, Share\&Charge is a platform that allows P2P transactions between $\mathrm{EV}$ drivers and the infrastructure owners. This is an Ethereum-based application, while the transaction is governed by a smart contract and a personal wallet of the user. Another solution based on V2G is proposed in the next section.

Another blockchain-based applications in the e-mobility are Innogy and Alliander.

Instead, another application of $\mathrm{BC}$ in the energy sector regard the use of the IoT for the real-time monitoring, metering and asset management. ElectricChain and SolarCoin cooperate for revenue in Solarcoin to each owner of a RES-based plant. The revenue is based on the produced energy that is monitored by IoT technology. Iot is also used in BC-based application that monitors in remote the status of electric devices, switching on/off them, depending on specific constraints fixed by the user. Hyperledger Fabric is instead used by Energy-Blockchain $\mathrm{Lab}$ and IBM into a platform that implements a carbon credit management. Also, CarbonX, that is instead based on Ethereum, proposes solution for $\mathrm{P} 2 \mathrm{P}$ carbon trading, soliciting a rationale use of the energy and putting in evidence the carbon footprint of their products.

Fig. 5 reports the amount, in per cent, of the BC-based applications or pilot-projects in different areas of the energy sector. It is worth noting to observe that the decentralized energy trading is the most investigated area, while the other areas have similar number of applications.

\section{Applications of $B C s$}

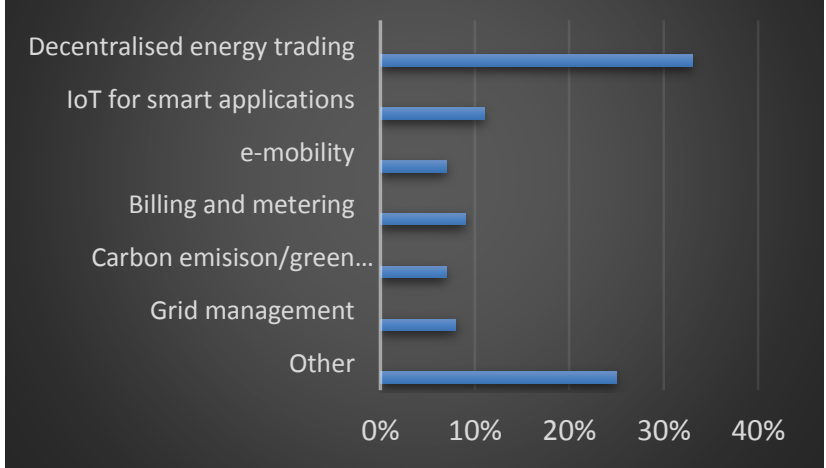

Fig. 5. Applications of BCs for different areas of the energy sector. 
Finally, Table I reports some pros and cons of the BC usege in the energy sector, while the advantages and disadvantages of the main features of the $\mathrm{BC}$ can be found in [16].

Table I. - Pros and cons of the BC usage in energy sector.

\begin{tabular}{|l|}
\multicolumn{1}{|c|}{ PROS } \\
\hline - Reduced transaction costs \\
- Reduced cost of utility bills \\
- Environmental sustainability \\
- Increased transparency for the stakeholders \\
- Promotion of the exchange of energy deriving from RES \\
- Increased penetration of the RES \\
\hline CONS \\
\hline - Increased complexity of the whole system \\
- Energy consumption \\
- BCalability is nowadays limited \\
\hline
\end{tabular}

\section{Proposal to reinforce the V2G}

As already said, the increase of the power deriving from RES (especially from PV and wind plants) has a significant impact on the grid, because of the unpredictability of the amount of the injected energy. For this reason, the strategy of including the storage systems in the electrical infrastructure is becoming more and more common. Nevertheless, these storage systems cannot be sized for the worst case, for sake of sustainability from the economic and financial point of view. So, the DSOs are searching for solutions able to manage and/or to mitigate the peak demand. A possible tool is considered the exploitation of the energy stored in the batteries of gridconnected vehicles, i.e. to apply the V2G strategy.

Nowadays, the application of V2G is based on an agreement between the owner of the EV and the DSO: the DSO can use the energy into the batteries and the owner receives revenues for this service. The agreement has to take into account several parameters (e.g. the degradation of the battery, due to the increased number of charge/discharge cycles), other than some technical constraints like the State Of Charge (SOC), the charging/discharging limits, the maximum time during which the vehicle is grid-connected, and so on. The constraint on the SOC must guarantee the correct and efficient operation of the batteries in the time, and then the maximum $\left(\mathrm{SOC}_{\max }\right)$ and minimum $\left(\mathrm{SOC}_{\min }\right)$ values must satisfy the following equation:

$$
S O C_{\min } \leq S O C(t) \leq S O C_{\max } \quad \forall t
$$

being $\operatorname{SOC}(\mathrm{t})$ the ratio between the current capacity at the instant $t$ and its nominal capacity:

$$
\operatorname{SOC}(\mathrm{t})=\frac{\mathrm{Q}(\mathrm{t})}{\mathrm{Q}_{\mathrm{n}}}
$$

SOC $(\mathrm{t})$ can be calculated based on both the previous value, $\mathrm{SOC}(\mathrm{t}-1)$, and the discharge current, $\mathrm{I}(\mathrm{t})$, by using the Coulomb counting method:

$$
\operatorname{SOC}(t)=\operatorname{SOC}(t-1)+\frac{I(t)}{Q_{n}} \Delta t
$$

Enhanced counting methods, considering other parameters (e.g. the life cycle of the battery), can be found in [17]-[19]. Instead, the capacity estimation of the energy available in the battery and the scheduling algorithm to charge an EV (before the owner disconnects it for the grid) are detailed in [20].

In order to stimulate the owners of EVs in the development of a BC that supports also the $\mathrm{V} 2 \mathrm{G}$, it needs to involve them in the validation of the new blocks, by providing a reliable selection mechanism of the validator. The validator can be chosen as the owner who has injected the maximum amount of energy, normalized to the nominal capacity of the battery, in the last thirty days:

$$
\max \sum_{k \leq 30}\left(\frac{E_{\text {inject }, k}}{Q_{n}}\right)
$$

where $E_{\text {inject, } k}$ is the total energy injected into the grid in the $k$-th day. This strategy can use the PoS as DCA, resulting energy-saving. It is worth noting that the normalized energy is intended to allow each owner of an $\mathrm{EV}$ to act as a $\mathrm{BC}$ validator, since the "total injected energy" parameter is not enough. Otherwise, only the owners of EVs equipped with higher capacity batteries would be selected as validators, but the most EVs are equipped with lower capacity batteries. Moreover, the choice of thirty days (that can be revised also in 1 week for specific cases) aims to retain the owners of EVs. In fact, the DSO needs to estimate the energy that will be injected into the grid in the next day and in the next week. Therefore, it is important that the potential validators inject energy into the grid almost every day. It is obvious that the validator must receive a reward to validate a new block, and this reward will consist of free recharge for the EV and it must be used in a limited time. This $\mathrm{BC}$ is public, energy-saving, permission-less and the privacy is governed by the same rules used for any owner of EVs participating to the V2G.

\section{Conclusion}

The current state of the art of the use of the BC technologies in the energy sector is diversified with respect to the DCAs (PoW, Pos, PBFT, etc.), privacy rule (known or unknown user and or validators), access (open-source or closed-source), privileges (permissionless or permissioned), sharing level of the DLT (totally or partially distributed), and so on. Moreover, the pilotprojects range from the energy trading to the e-mobility, from the grid management to the carbon emissions. This implies that there are not consolidated approaches and strategies for the use of a specific BC for each specific area of the energy sector. This consideration is valid also for the characteristic features: for example, there is not a consolidated position if the permission-less approach is 
better than the permissioned one for a specific topic. Therefore, extensive research possibilities are available. This paper has proposed an energy-saving, permission-less $\mathrm{BC}$ that allows every owner of EVs to validate a new block, if the normalized energy injected into the grid in the last month is higher than the energy injected by other owners. This application has been considered for a V2G application.

\section{References}

[1] A. Massi Pavan, S. Vergura, A. Mellit, V. Lughi, "Explicit empirical model for photovoltaic devices. Experimental validation", Solar Energy, Vol. 155, October 2017, pp. 647653. ISSN 0038-092X.

[2] S. Vergura, A. Massi Pavan, "On the photovoltaic explicit empirical model: operations along the current-voltage curve", IEEE-ICCEP 2015 International Conference on Clean Electrical Power, Taormina, Italy, June 16-18, 2015.

[3] Eid C, Codani P, Perez Y, Reneses J, Hakvoort R. Managing electric flexibility from Distributed Energy Resources: a review of incentives for market design. Renewable Sustainable Energy Reviews, 2016;64:237-47.

[4] Luo X, Wang J, Dooner M, Clarke J. Overview of current development in electrical energy storage technologies and the application potential in power system operation. Applied Energy, 2015;137:511-36.

[5] Zhou S, Brown MA. Smart meter deployment in Europe: a comparative case study on the impacts of national policy schemes. Journal of Cleaner Production, 2017;144:22-32.

[6] Berka AL, Creamer E. Taking stock of the local impacts of community owned renewable energy: a review and research agenda. Renewable and Sustainable Energy Reviews, 2017;82(Part (3)):3400-19.

[7] Marnay C, Chatzivasileiadis S, Abbey C, Iravani R, Joos G, Lombardi $\mathrm{P}$, et al., Microgrid evolution roadmap. In: Proceedings of the 2015 International Symposium on Smart Electric Distribution Systems Technologies (EDST), IEEE, 2015, pp. 139-44.

[8] Ton DT, Smith MA. The US Department of Energy's microgrid initiative. The Electricity Journal, 2012;25(8):84-94.

[9] S. Vergura, F. Marino, "Quantitative and Computer Aided Thermography-based Diagnostics for PV Devices: Part I -
Framework", IEEE Journal of Photovoltaics (2017), Vol. 7, Issue 3, pp. 822-827.

[10] S. Vergura, M. Colaprico, M. F. de Ruvo, F. Marino, “A Quantitative and Computer Aided Thermography-based Diagnostics for PV Devices: Part II - Platform and Results”, IEEE Journal of Photovoltaics (2017), Vol. 7, Issue 1, pp. 237-243.

[11] G. Acciani, G.B. Simione, S. Vergura, "Thermographic Analysis of Photovoltaic Panels", Renewable Energy \& Power Quality Journal (2010), No.8.

[12] G. Acciani, O. Falcone, S. Vergura, "Analysis of the thermal heating of poly-Si and a-Si photovoltaic cell by means of Fem", Renewable Energy \& Power Quality Journal (2010), No.8.

[13] F. Rahimi, "Transactive energy framework for bilateral energy imbalance management," in Proc. GridWise Architecture Council Meet., Dec. 2013., [Online] Available: https://www.gridwiseac.org/ Accessed on: May 14, 2018

[14] Mylrea M. Gourisetti SNG. Blockchain for smart grid resilience: Exchanging distributed energy at speed, scale and security. In: Proceedings of the Resilience Week (RWS) 2017, IEEE, 2017, pp. 18-23.

[15] Mengelkamp E, Gärttner J, Rock K, Kessler S, Orsini L, Weinhardt C. Designing microgrid energy markets A case study: the Brooklyn Microgrid. Applied Energy, 2018; 210:870-80.

[16] https://issuu.com/bdew_ev/docs/studie-blockchain-engl (visited website in January 2020).

[17] X. Jiale, M. Jiachen, B. Kun, Xie, Jiale, Enhanced Coulomb Counting Method for State-of-Charge Estimation of Lithium-ion Batteries based on Peukert's Law and Coulombic Efficiency, Journal of Power Electronics, Vol. 18 Issue 3, pp. 910-922, 2018.

[18] C. Zhang, K. Li, L. Pei, and C. Zhu, An integrated approach for real-time model-based state-of-charge estimation of lithium-ion batteries, J. Power Sources, Vol. 283, pp. 24-36, Jun. 2015.

[19] Y. He, X. Liu, C. Zhang, and Z. Chen, A new model for State-of-Charge (SOC) estimation for high-power Li-ion batteries, Applied Energy, Vol. 101, pp. 808-814, Jan. 2013.

[20] P. Du, N. Lu, Energy storage for smart grids, 1st ed., Elsevier, pp. 249-274, 2015. 